\title{
Interaction-induced topological superconductivity in antiferromagnet-superconductor junctions
}

\author{
Senna S. Luntama, Päivi Törmä®i, and Jose L. Lado @* \\ Department of Applied Physics, Aalto University, 00076 Aalto, Espoo, Finland
}

(Received 14 November 2020; revised 26 January 2021; accepted 8 February 2021; published 5 March 2021)

\begin{abstract}
We predict that junctions between an antiferromagnetic insulator and a superconductor provide a robust platform to create a one-dimensional topological superconducting state. Its emergence does not require the presence of intrinsic spin-orbit coupling nor noncollinear magnetism, but arises solely from repulsive electronic interactions on interfacial solitonic states. We demonstrate that a topological superconducting state is generated by repulsive interactions at arbitrarily small coupling strength, and that the size of the topological gap rapidly saturates to one of the parent trivial superconductor. Our results put forward antiferromagnetic insulators as a new platform for interaction-driven topological superconductivity.
\end{abstract}

DOI: 10.1103/PhysRevResearch.3.L012021

The search for topological superconductors has been one of the most active areas in condensed matter physics in recent years [1-19]. These systems, pursued in particular for the emergence of Majorana zero modes, represent one of the potential solid state platform for the implementation of topological quantum computing [20,21]. Due to their elusive nature, topological superconductors are often artificially engineered. A variety of platforms have been proposed and demonstrated for this purpose [17,22,23], generically relying on a combination of conventional $s$-wave superconductivity, ferromagnetism, and strong spin-orbit coupling [7-10,15,24,25].

While ferromagnets have played a central role for artificial topological superconductivity, antiferromagnetic insulators have been overlooked for this purpose. Recently, antiferromagnets have attracted a great amount of attention due to their unique properties for spintronics [26-30] and for creating novel types of topological matter [31-37]. Ferromagnetism efficiently lifts Kramer's degeneracy, a process heavily detrimental for spin-singlet superconductivity. Antiferromagnetism, in comparison, does not lift Kramer's degeneracy between opposite spins in the absence of spin-orbit coupling, a feature that could potentially make antiferromagnetism more compatible with spin-singlet superconductivity [38-45].

Here we show that two-dimensional topologically trivial antiferromagnetic insulators provide a platform to design onedimensional topological superconductivity. In our proposal, spin-orbit coupling effects are not necessary for topological superconductivity to appear, nor a fine tuning between the different components of the system. In contrast, we show that long-range interactions alone give rise to a nontrivially gapped

\footnotetext{
*jose.lado@aalto.fi
}

Published by the American Physical Society under the terms of the Creative Commons Attribution 4.0 International license. Further distribution of this work must maintain attribution to the author(s) and the published article's title, journal citation, and DOI. state hosting Majorana excitations, and that the interactioninduced gap opening is topological irrespective of details. We demonstrate that the robustness of this unique state stems from the solitonic nature of the emergent excitations at the interface, in which interaction-induced gap opening unavoidably gives rise to a topological superconducting state. Our results put forward antiferromagnet-superconductor junctions as a robust platform to engineer interaction-induced topological superconductivity.

Our system consists of a junction between a conventional $s$-wave superconductor and antiferromagnetic insulator, as shown in Fig. 1(a). To model this system we take a Hamiltonian in the honeycomb lattice of the form

$$
\mathcal{H}=\mathcal{H}_{\text {kin }}+\mathcal{H}_{\mathrm{AF}}+\mathcal{H}_{\mathrm{SC}}+\mathcal{H}_{\text {int }},
$$

where

$$
\begin{gathered}
\mathcal{H}_{\mathrm{kin}}=t \sum_{\langle i j\rangle, s} c_{i, s}^{\dagger} c_{j, s}+\sum_{i, s} \mu\left(\mathbf{r}_{i}\right) c_{i, s}^{\dagger} c_{i, s}, \\
\mathcal{H}_{\mathrm{AF}}=\sum_{i, s} m_{\mathrm{AF}}\left(\mathbf{r}_{i}\right) \tau_{i, i}^{z} \sigma_{s, s}^{z} c_{i, s}^{\dagger} c_{i, s}, \\
\mathcal{H}_{\mathrm{SC}}=\sum_{i} \Delta\left(\mathbf{r}_{i}\right) c_{i, \uparrow} c_{i, \downarrow}+\text { H.c. }, \\
\mathcal{H}_{\mathrm{int}}=V_{1} \sum_{\langle i j\rangle}\left(\sum_{s} c_{i, s}^{\dagger} c_{i, s}\right)\left(\sum_{s} c_{j, s}^{\dagger} c_{j, s}\right) \\
+V_{2} \sum_{\langle\langle i j\rangle\rangle}\left(\sum_{s} c_{i, s}^{\dagger} c_{i, s}\right)\left(\sum_{s} c_{j, s}^{\dagger} c_{j, s}\right),
\end{gathered}
$$

where $c_{i, s}^{\dagger}$ is the fermionic creation operator for site $i$ and for spin $s, \sigma^{z}$ denotes the spin Pauli matrix, $\tau^{z}$ is the sublattice Pauli matrix, \langle\rangle is the first neighbors, and $\langle\langle\rangle\rangle$ is the second neighbors. Taking that the interface between the antiferromagnet and the superconductor is located at $\mathbf{r}=(x, 0,0)$ we take $\Delta(\mathbf{r})=\frac{\Delta}{2}[1-\operatorname{sgn}(y)] \mu(\mathbf{r})=\frac{\mu}{2}[1-\operatorname{sgn}(y)]$ and $m_{\mathrm{AF}}(\mathbf{r})=$ $\frac{m_{\mathrm{AF}}}{2}[1+\operatorname{sgn}(y)][46]$. The repulsive interaction term of Eq. (5) is solved at the mean-field level including the usual meanfield decouplings $\mathcal{H}_{\mathrm{int}} \approx \mathcal{H}^{\mathrm{MF}}=\sum \chi_{i j s s^{\prime}} c_{i, s}^{\dagger} c_{j, s^{\prime}}$ with $\chi_{i j s s^{\prime}}$ the 
(a)
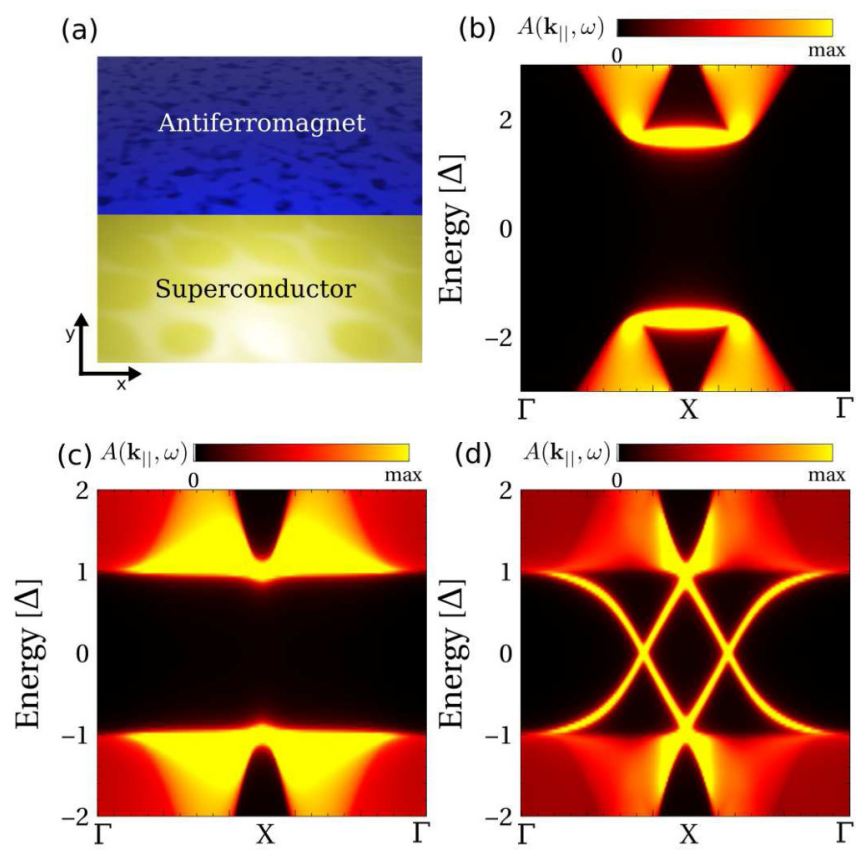

(e) $A(\mathbf{r}, \omega=0)$

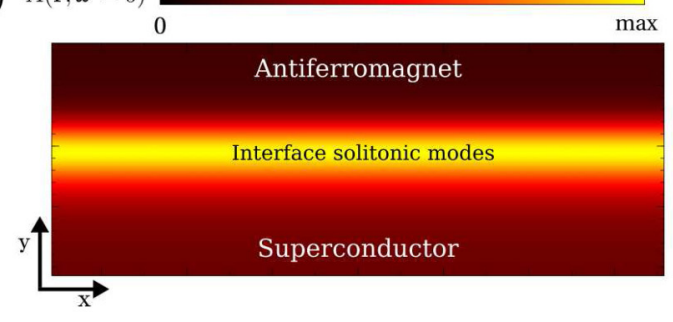

FIG. 1. (a) A sketch of the two-dimensional antiferromagnet (AF) and superconductor (SC) forming a one-dimensional AF-SC interface. The spectral function at the surface of the AF (b), at the surface of the SC (c), and at the interface between AF and SC (d) as given by our model Hamiltonian (1) in a honeycomb lattice. (e) The spatial distribution of the interfacial modes. Here we chose $\Delta=0.3 t$, $m_{\mathrm{AF}}=0.5 t, \mu=t$, and $V_{1}=V_{2}=0$.

self-consistent mean-field parameters [47] . On-site interactions are incorporated in $m_{\mathrm{AF}}(\mathbf{r})$ and $\Delta(\mathbf{r})$ at the mean-field level.

It is instructive to examine the electronic band structure in the absence of interactions and in the absence of an interface. Let us consider a semi-infinite slab in the $y$ direction, having translational symmetry in the $x$ direction as depicted in Fig. 1(a). For that geometry we compute the momentum-resolved spectral function at the edge $A\left(\mathbf{k}_{\|}, \omega\right)=$ $-\frac{1}{\pi} \operatorname{Im}\left[\omega-\mathcal{H}\left(\mathbf{k}_{||}\right)+i 0^{+}\right]^{-1}$ using the Dyson formalism [48]. For both isolated superconductor and antiferromagnet, the surface spectral function presents a gap, as shown in in Figs. 1(b) and 1(c), that simply stems from the gapped topologically trivial band structure. In the case of the superconductor the gap is controlled by $\Delta$, whereas in the antiferromagnet, the gap is determined by $m_{\mathrm{AF}}$. In stark contrast, when the antiferromagnet and superconductor are joined together, a new branch of interfacial modes appear as shown in Fig. 1(d). By computing the spectral function in real space at zero energy $A(\mathbf{r}, \omega=0)$ it is clearly seen that the new branch is heavily localized at the junction between the superconductor and the antiferromagnet. We have verified that for different values of the superconducting and antiferromagnet order parameters, zero modes emerge as long as the order parameters are not substantially bigger than the typical bandwidth.

The emergence of the interfacial zero modes can be rationalized from a low energy model for the honeycomb lattice [49-53]. For the following analytic derivation, it is convenient to take $\mu=0$ so that the full antiferromagnet-superconductor can be described with a generalized Dirac equation at the $K$ point of the honeycomb lattice [54]. The low energy excitations can be captured by an effective model around the valleys $\mathcal{V}^{z}= \pm 1$, and we will focus first on taking the momentum parallel to the interface $p_{x}=0$. By defining the Nambu spinor $\Psi^{\dagger}=\left(c_{A, \uparrow, \mathbf{k}}^{\dagger}, c_{B, \uparrow, \mathbf{k}}^{\dagger}, c_{A, \downarrow,-\mathbf{k}}, c_{B, \downarrow,-\mathbf{k}}\right)$, the Hamiltonian in the electron-up/hole-down sector $(\Uparrow)$ can be written as $\mathcal{H}\left(p_{x}=\right.$ $\left.0, p_{y}\right)_{\kappa}=\frac{1}{2} \Psi^{\dagger} H_{\kappa} \Psi$ with

$$
H_{\kappa}=\left(\begin{array}{cccc}
m_{\mathrm{AF}}(\mathbf{r}) & p_{y} & \Delta(\mathbf{r}) & 0 \\
p_{y} & -m_{\mathrm{AF}}(\mathbf{r}) & 0 & \Delta(\mathbf{r}) \\
\Delta(\mathbf{r}) & 0 & m_{\mathrm{AF}}(\mathbf{r}) & -p_{y} \\
0 & \Delta(\mathbf{r}) & -p_{y} & -m_{\mathrm{AF}}(\mathbf{r})
\end{array}\right) .
$$

The spectrum of this effective model is gapped at $y \pm \infty$, as expected from its asymptotic antiferromagnet/superconductor gap. However, a zero energy mode $H\left|\psi_{\Uparrow}\right\rangle=0$ at the interface can be always built, taking the functional form $\psi_{\Uparrow}^{\dagger}=$ $e^{-\int_{0}^{y}\left[\Delta\left(y^{\prime}\right)-m_{\mathrm{AF}}\left(y^{\prime}\right)\right] d y^{\prime}}\left(c_{A, \uparrow}^{\dagger}+i c_{B, \uparrow}^{\dagger}-i c_{A, \downarrow}-c_{B, \downarrow}\right)$. The nature of this zero mode is analogous to the Jackiw-Rebbi soliton [49], and therefore can be understood as an antiferromagnet-superconducting soliton. The complementary electron-down/hole-up $(\Downarrow)$ sector of the Hamiltonian will therefore also host a zero mode that we label as $\psi_{\Downarrow}$. Away from the point $p_{x}=0$, the previous state acquires a finite dispersion given by first order perturbation theory $v_{F} p_{x}=$ $\left\langle\psi_{\Uparrow}|\mathcal{H}| \psi_{\Uparrow}\right\rangle$. As a result, close to the $K$ points two branches of zero modes appear, giving rise to the effective low energy Hamiltonian

$$
H\left(p_{x}\right)=\sum_{\kappa} v_{F} p_{x} \mathcal{V}_{\kappa, \kappa}^{z}\left[\psi_{\Uparrow, \kappa, p_{x}}^{\dagger} \psi_{\Uparrow, \kappa, p_{x}}-\psi_{\Downarrow, \kappa, p_{x}}^{\dagger} \psi_{\Downarrow, \kappa, p_{x}}\right],
$$

where $\kappa$ runs over the two valleys. It is interesting to note that the four modes are not independent, but they are related by electron-hole symmetry operator $\Xi=\theta^{y} \sigma^{y} \mathcal{C}$ with $\theta^{y}$ the Nambu Pauli matrix and $\mathcal{C}$ the complex conjugation as $\Xi^{-1} \psi_{\Uparrow,+1, p_{x}} \Xi=\psi_{\Downarrow,-1,-p_{x}}$ due to the built-in Nambu electron-hole symmetry of the Hamiltonian. Therefore, the Hamiltonian Eq. (7) hosts only two physical degrees of freedom, each one propagating in opposite directions, realizing an effective spinless one-dimensional model. These singlydegenerate channels are analogous to quantum Hall edge states [15], and helical channels in topological insulators [7], states that provide a starting point for engineering a topological superconducting gap. Remarkably in our case, as will be shown below, the solitonic gapless channels will open up a topological superconducting gap once electron-electron interaction effects are included.

Let us now move on to consider the impact of long-range electronic interactions in the solitonic modes. For computational convenience we now perform our calculations in 

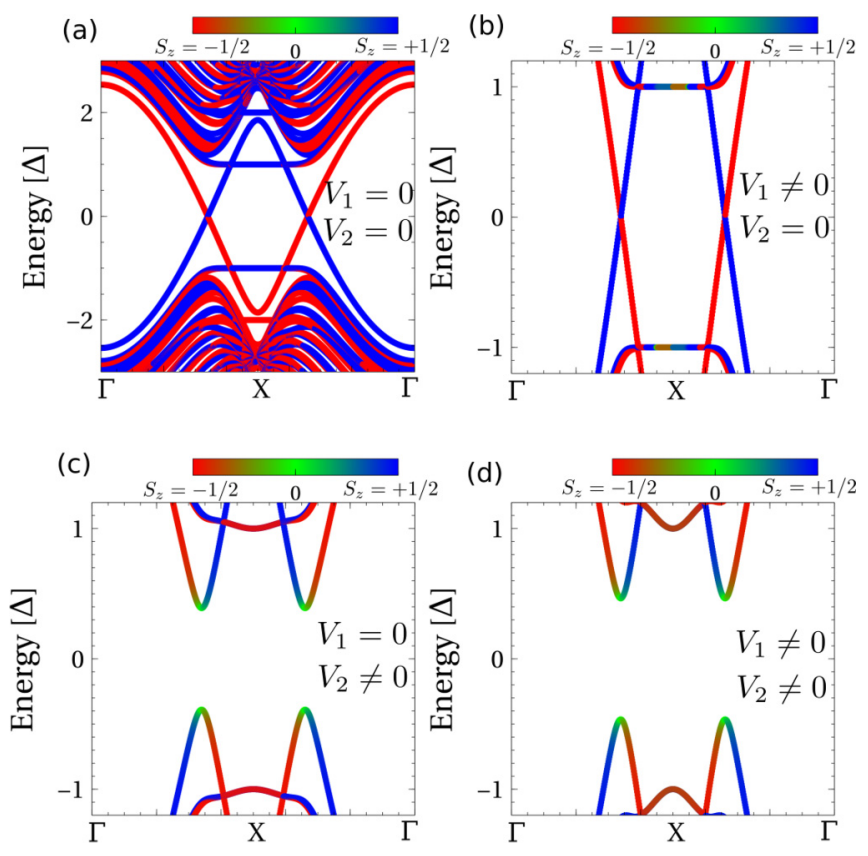

FIG. 2. (a) Noninteracting bands in a ribbon geometry. First neighbor interactions do not lead to a gap (b), whereas second neighbor interactions drive a gap opening (c). When both first and second neighbor interactions are present the gap remains. The parameters are $V_{1}=t$ in (b), $V_{2}=1.7 t$ in (c), $V_{1}=t, V_{2}=2 t$ in (d), and $m_{\mathrm{AF}}=0.8 t, \Delta=0.4 t$ in (a) $-(\mathrm{d})$.

ribbons of finite width in the $x$ direction, in which we take the transverse direction wide enough to avoid finite-size effects. The previous gapless interface modes of Fig. 1(d) and derived in Eq. (7) appear in this ribbon geometry as shown in Fig. 2(a), where $S_{z}=\frac{1}{2}\left\langle\sum_{n, s} \sigma_{s, s}^{z} c_{n, s}^{\dagger} c_{n, s}\right\rangle_{\Psi_{k}}$ with $\Psi_{k}$ the eigenstate. It is shown that in the absence of interactions, the sectors $S_{z}= \pm 1 / 2$ are fully decoupled, stemming from the $U(1)$-spin symmetry of the Hamiltonian. With this lattice model we now explore the impact of electronic interactions by solving self-consistently Eq. (1). Note that the interactions apply both along the interface and across it. We start by considering only first neighbor interactions, taking $V_{2}=0$. In this situation, a gap does not open even when $V_{1}$ is increased, as shown in Fig. 2(b). We now move on to the case of $V_{2}$, taking first $V_{1}=0$. As observed in Fig. 2(c), it is clearly seen that now a gap opens up. This behavior also takes place when $V_{1}$ is taken to be nonzero, see Fig. 2(d). As a result, second neighbor interactions are the only interaction capable of opening up a gap on the topological interface modes, whose magnitude is marginally affected by the first neighbor interactions.

The emergence of a gap opening driven by electronic interactions raises the question of potential nontrivial topological properties. From the point of view of the effective low energy model, interactions create an effective term in Eq. (7) of the form $H^{\mathrm{MF}} \sim\left\langle\Psi_{\Uparrow} \Psi_{\Downarrow}^{\dagger}\right\rangle \Psi_{\Uparrow}^{\dagger} \Psi_{\Downarrow}+$ H.c. It is interesting to note that due to the solitonic functional form of $\Psi_{\Uparrow}$ and $\Psi_{\Downarrow}$ and their relation via electron-hole symmetry, the gap $\left(\propto\left\langle\Psi_{\Uparrow} \Psi_{\Downarrow}^{\dagger}\right\rangle\right)$ created is odd with respect to $\kappa$, the valley index, suggesting the emergence of an effective topological superconducting state. To verify the nontrivial topological nature of the interaction-driven gapped state, we compute both its $Z_{2}$ (a)

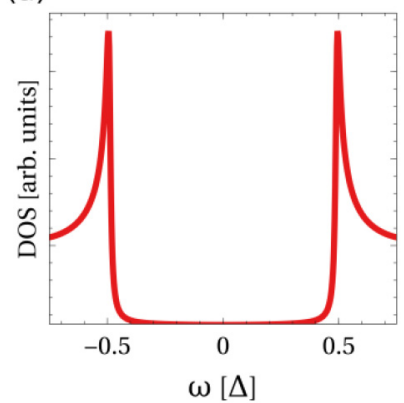

(c)
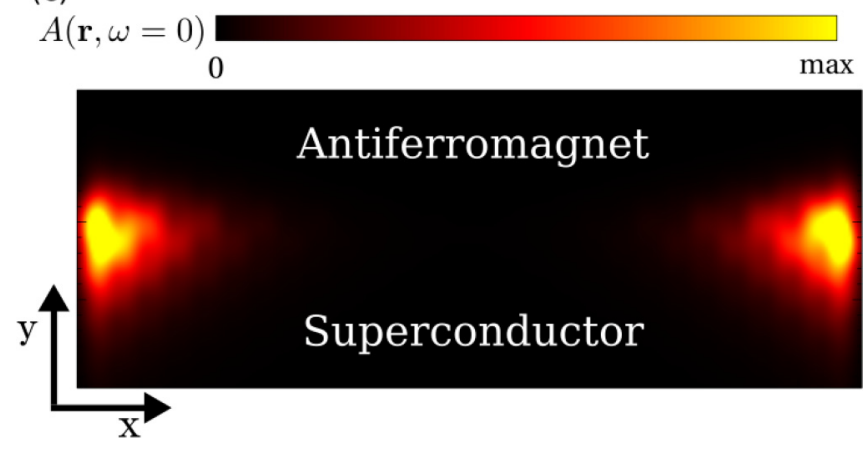

FIG. 3. (a) Spectral function in the bulk in the presence of interactions, and (b) at the edge showing the emergence of a zero Majorana mode. (c) The spectral function at $\omega=0$ for a finite junction, featuring edge zero Majorana modes. We used now $\Delta=0.4 t$, $m_{\mathrm{AF}}=0.8 t, V_{1}=t$, and $V_{2}=2 t$.

topological invariant $[1,55]$ and surface spectral function. We revealed that the gapped system has a topologically nontrivial $Z_{2}$ invariant, signaling the existence of a topological superconducting state. This is further verified when computing the density of states at the edge of the interface in a ribbon that spans from $x=0$ to $x=\infty$, as shown in Fig. 3(a). The edge of the system hosts a zero-mode resonance associated with the unpaired Majorana stemming from the nontrivial electronic structure. This is contrasted with the finite gap present in the bulk of the system shown in Fig. 3(b). The localization of the zero mode can also be seen when computing the spectral function for $\omega=0$, Fig. 3(c).

Let us now move on to look at the impact of long-range interactions, and in particular at the interplay between the first and second neighbor interactions at the mean-field level. For the sake of simplicity in the following discussion we will only consider effects that appear by means of a mean-field decoupling of Eq. (5), without considering beyond mean-field effects or additional $t-J$ contributions. At the mean-field level, the interaction term of Eq. (5) can give rise to two potential effects: first, interaction induced hoppings and second, symmetry broken states such as charge density waves. In the weak coupling regime considered here, only interaction-induced hopping terms arise. In particular, the time-reversal symmetric and spin-dependent part of $\chi_{i j s s^{\prime}}$ yield an effective spin and spatially dependent synthetic spin-orbit coupling term of the Kane-Mele form [7,56]. This interaction-induced term creates spin mixing in the solitonic modes, opening up a topological gap. 
(a)

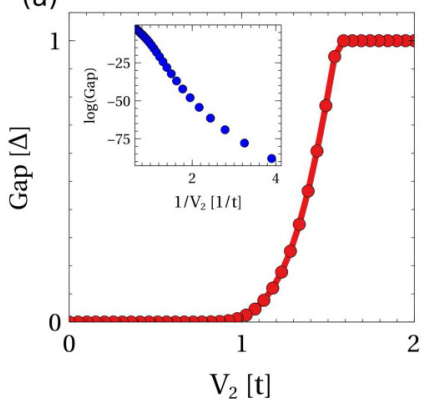

(c)

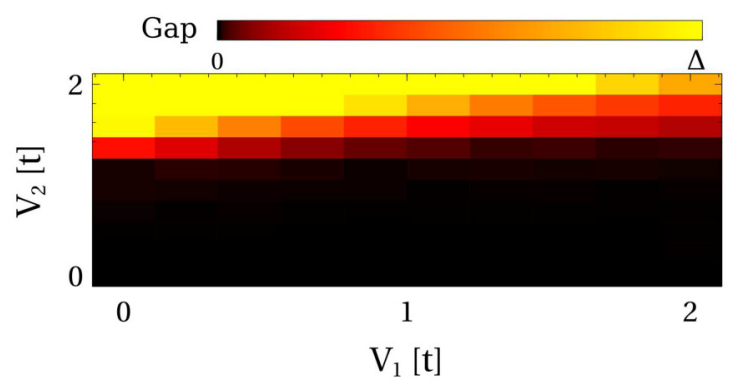

FIG. 4. (a) Evolution of the topological gap with the electronelectron interaction: (a) As a function of $V_{2}$ taking $V_{1}=0$ and (b) as a function of $V_{1}$ taking $V_{2}=2 t$. (c) The topological gap as a function of the two electronic interactions $V_{1}$ and $V_{2}$, highlighting that only the second neighbor interaction opens up a gap. We took $\Delta=0.2 t$ and $m_{\mathrm{AF}}=0.4 t$.

The interplay of first and second neighbor interactions can be easily rationalized within this language. From the mean-field point of view, first neighbor interactions can give rise to interaction induced Rashba spin-orbit coupling terms [57], whereas second neighbor interactions can give rise to interaction-induced Kane-Mele spin-orbit coupling [58]. However, due to the valley polarized nature of the solitonic modes, interaction induced Rashba-spin-orbit coupling does not open up a gap in them [57], whereas Kane-Mele like spin-orbit [58] can create a gap. As a result, second neighbor interactions are the only ones capable of an interactioninduced gap opening in the system. In contrast, the effect of the first neighbor interactions is to simply create a Fermi velocity renormalization $[59,60]$ increasing the kinetic energy of the solitonic modes, yet without any competing mechanism for gap opening.

It is crucial to understand whether the gap opening requires a finite minimum value of interaction strength. We investigate this by taking the first neighbor interaction $V_{1}=0$, and looking at the topological gap as a function of the repulsive second neighbor interaction $V_{2}$. It is clearly observed that the topological gap becomes stronger as $V_{2}$ is increased, without the existence of a critical value for the transition [Fig. 4(a)]. In particular, a logarithmic plot of the gap [inset of Fig. 4(a)] at small coupling strength reveals that the topological gap $\delta$ follows an exponential dependence $\delta \sim e^{-\frac{v_{F}}{V_{2}}}$ [61]. Interestingly, whereas exponential dependencies of that form are typical for superconducting instabilities driven by attractive interactions [62], in our present case interactions are actually repulsive. This behavior stems from the projection of the interactions in the low energy solitonic model of Eq. (7), driving a topological phase transition at arbitrarily small couplings. At large coupling strengths $V_{2}$, the topological gap saturates to the gap of the superconductor. This behavior should be contrasted with the other schemes proposed for topological superconductivity, in which the topological gap is usually substantially smaller than the original superconductor gap. This saturation of the topological gap can be ascribed to the absence of competition between the superconductor and the antiferromagnet. Including finite first neighbor interactions $V_{1}$ keeps the picture qualitatively unchanged, yet with a slightly renormalized topological gap [Fig. 4(b)]. The interplay between $V_{1}$ and $V_{2}$ shown in Fig. 4(c) shows that, whereas $V_{2}$ opens the topological gap, $V_{1}$ leaves the system gapless or slightly renormalizes the topological gap. Finally, we note that imperfections and disorder are known to potentially impact topological superconductors by limiting the localization length and reducing the topological gap [63-65]. We verified that the phenomenology presented above is resilient towards Anderson disorder and happens for generic AF-SC interfaces [66]. Disorder slightly decreases the topological gap, yet without qualitatively impacting our results.

Finally, we address the potential experimental realization of our proposal. For a solid-state realization, no specific requirements are necessary for the superconductor besides conventional $s$-wave pairing, as realized in $\mathrm{NbSe}_{2}$. The fundamental requirement is having a two-dimensional honeycomb antiferromagnetic insulator [67], as its electronic structure is expected to have the gapped Dirac points required for the emergence of the topological solitonic modes. Within van der Waals materials, trihalides host a magnetic honeycomb lattice [68], and in particular antiferromagnetic strained trihalides $[69,70]$ would be suitable for our proposal. This pathway would require creating superconductor/antiferromagnet devices with those strained van der Waals materials. Within oxides, thin films of $\operatorname{InCu}_{2 / 3} \mathrm{~V}_{1 / 3} \mathrm{O}_{3}$ [71] or $\beta-\mathrm{Cu}_{2} \mathrm{~V}_{2} \mathrm{O}_{7}$ [72] has the required antiferromagnetic honeycomb lattice. For this possibility, a single layer of the bulk oxide should be epitaxially grown. Generic two-dimensional antiferromagnetic insulators hosting Dirac points in their normal state [73] would be suitable materials for our proposal, whose specific $V_{1}, V_{2}$ parameters can be inferred by first-principles methods [74-78]. Finally, future ultracold atom setups [79] are potential platforms for the realization of our model, as honeycomb structures [80], antiferromagnetic correlations [81], long-range interactions [82-84], and $s$-wave correlations [85] in the normal state have been separately demonstrated. Interactions can be tuned from attractive to repulsive by magnetic fields; spatially dependent fields could be one way of creating the AF-SC interface, once superfluid correlations in a lattice have been reached.

To summarize, we have shown that an interface between a topologically trivial two-dimensional superconductor and antiferromagnetic insulator gives rise to a one-dimensional solitonic gas. Upon introduction of repulsive long-range interactions, we have demonstrated that a topological gap gets generated, giving rise to Majorana zero energy modes. The emergence of topological superconductivity appears in the absence of intrinsic spin-orbit coupling and is driven by repulsive Coulomb interactions. We showed that the topological gap appears at arbitrarily small interactions, and rapidly satu- 
rates to the gap of the parent superconductor, in stark contrast with conventional proposals involving competition between ferromagnetism and superconductivity. Our results propose a new mechanism to generate topological superconductivity based on interacting solitons, putting forward antiferromagnetic insulators as a potential materials platform for Majorana physics.
We acknowledge the computational resources provided by the Aalto Science-IT project. J.L.L. acknowledges financial support from the Academy of Finland Projects No. 331342 and No. 336243. P.T. acknowledges support by the Academy of Finland under Projects No. 303351, No. 307419, and No. 327293.
[1] A. Yu. Kitaev, Unpaired Majorana fermions in quantum wires, Phys. Usp. 44, 131 (2001).

[2] J. Alicea, New directions in the pursuit of Majorana fermions in solid state systems, Rep. Prog. Phys. 75, 076501 (2012).

[3] C. W. J. Beenakker, Search for Majorana fermions in superconductors, Annu. Rev. Condens. Matter Phys. 4, 113 (2013).

[4] C. Beenakker and L. Kouwenhoven, A road to reality with topological superconductors, Nat. Phys. 12, 618 (2016).

[5] M. T. Deng, C. L. Yu, G. Y. Huang, M. Larsson, P. Caroff, and H. Q. Xu, Anomalous zero-bias conductance peak in a Nb-InSb nanowire-Nb hybrid device, Nano Lett. 12, 6414 (2012).

[6] A. D. K. Finck, D. J. Van Harlingen, P. K. Mohseni, K. Jung, and X. Li, Anomalous Modulation of a Zero-Bias Peak in a Hybrid Nanowire-Superconductor Device, Phys. Rev. Lett. 110, 126406 (2013).

[7] L. Fu and C. L. Kane, Superconducting Proximity Effect and Majorana Fermions at the Surface of a Topological Insulator, Phys. Rev. Lett. 100, 096407 (2008).

[8] J. Röntynen and T. Ojanen, Topological Superconductivity and High Chern Numbers in 2D Ferromagnetic Shiba Lattices, Phys. Rev. Lett. 114, 236803 (2015).

[9] P. Hosur, P. Ghaemi, R. S. K. Mong, and A. Vishwanath, Majorana Modes at the Ends of Superconductor Vortices in Doped Topological Insulators, Phys. Rev. Lett. 107, 097001 (2011).

[10] R. M. Lutchyn, J. D. Sau, and S. Das Sarma, Majorana Fermions and a Topological Phase Transition in Semiconductor-Superconductor Heterostructures, Phys. Rev. Lett. 105, 077001 (2010).

[11] J.-P. Xu, C. Liu, M.-X. Wang, J. Ge, Z.-L. Liu, X. Yang, Y. Chen, Y. Liu, Z.-A. Xu, C.-L. Gao, D. Qian, F.-C. Zhang, and J.F. Jia, Artificial Topological Superconductor by the Proximity Effect, Phys. Rev. Lett. 112, 217001 (2014).

[12] G. Xu, B. Lian, P. Tang, X.-L. Qi, and S.-C. Zhang, Topological Superconductivity on the Surface of Fe-Based Superconductors, Phys. Rev. Lett. 117, 047001 (2016).

[13] Y. Asano and Y. Tanaka, Majorana fermions and odd-frequency cooper pairs in a normal-metal nanowire proximity-coupled to a topological superconductor, Phys. Rev. B 87, 104513 (2013).

[14] Q. L. He, L. Pan, A. L. Stern, E. C. Burks, X. Che, G. Yin, J. Wang, B. Lian, Q. Zhou, E. S. Choi, K. Murata, X. Kou, Z. Chen, T. Nie, Q. Shao, Y. Fan, S.-C. Zhang, K. Liu, J. Xia, and K. L. Wang, Chiral Majorana fermion modes in a quantum anomalous Hall insulator-superconductor structure, Science 357, 294 (2017).

[15] F. Finocchiaro, F. Guinea, and P. San-Jose, Topological $\pi$ Junctions from Crossed Andreev Reflection in the Quantum Hall Regime, Phys. Rev. Lett. 120, 116801 (2018).
[16] M. Ruby, F. Pientka, Y. Peng, F. von Oppen, B. W. Heinrich, and K. J. Franke, End States and Subgap Structure in ProximityCoupled Chains of Magnetic Adatoms, Phys. Rev. Lett. 115, 197204 (2015).

[17] S. Nadj-Perge, I. K. Drozdov, J. Li, H. Chen, S. Jeon, J. Seo, A. H. MacDonald, B. A. Bernevig, and A. Yazdani, Observation of Majorana fermions in ferromagnetic atomic chains on a superconductor, Science 346, 602 (2014).

[18] S. M. Albrecht, A. P. Higginbotham, M. Madsen, F. Kuemmeth, T. S. Jespersen, J. Nygård, P. Krogstrup, and C. M. Marcus, Exponential protection of zero modes in Majorana islands, Nature (London) 531, 206 (2016).

[19] M. T. Deng, S. Vaitiekenas, E. B. Hansen, J. Danon, M. Leijnse, K. Flensberg, J. Nygård, P. Krogstrup, and C. M. Marcus, Majorana bound state in a coupled quantum-dot hybrid-nanowire system, Science 354, 1557 (2016).

[20] J. Alicea, Y. Oreg, G. Refael, F. von Oppen, and M. P. A. Fisher, Non-Abelian statistics and topological quantum information processing in 1D wire networks, Nat. Phys. 7, 412 (2011).

[21] R. S. K. Mong, D. J. Clarke, J. Alicea, N. H. Lindner, P. Fendley, C. Nayak, Y. Oreg, A. Stern, E. Berg, K. Shtengel, and M. P. A. Fisher, Universal Topological Quantum Computation from a Superconductor-Abelian Quantum Hall Heterostructure, Phys. Rev. X 4, 011036 (2014).

[22] V. Mourik, K. Zuo, S. M. Frolov, S. R. Plissard, E. P. A. M. Bakkers, and L. P. Kouwenhoven, Signatures of Majorana fermions in hybrid superconductor-semiconductor nanowire devices, Science 336, 1003 (2012).

[23] S. Kezilebieke, M. Nurul Huda, V. Vaňo, M. Aapro, S. C. Ganguli, O. J. Silveira, S. Głodzik, A. S. Foster, T. Ojanen, and P. Liljeroth, Topological superconductivity in a designer ferromagnet-superconductor van der Waals heterostructure, Nature (London) 588, 424 (2020).

[24] Y. Oreg, G. Refael, and F. von Oppen, Helical Liquids and Majorana Bound States in Quantum Wires, Phys. Rev. Lett. 105, 177002 (2010).

[25] J. D. Sau, R. M. Lutchyn, S. Tewari, and S. Das Sarma, Generic New Platform for Topological Quantum Computation Using Semiconductor Heterostructures, Phys. Rev. Lett. 104, 040502 (2010).

[26] T. Jungwirth, X. Marti, P. Wadley, and J. Wunderlich, Antiferromagnetic spintronics, Nat. Nanotechnol. 11, 231 (2016).

[27] V. Baltz, A. Manchon, M. Tsoi, T. Moriyama, T. Ono, and Y. Tserkovnyak, Antiferromagnetic spintronics, Rev. Mod. Phys. 90, 015005 (2018)

[28] D.-F. Shao, G. Gurung, S.-H. Zhang, and E. Y. Tsymbal, Dirac Nodal Line Metal for Topological Antiferromagnetic Spintronics, Phys. Rev. Lett. 122, 077203 (2019). 
[29] W. Zhang, M. B. Jungfleisch, W. Jiang, J. E. Pearson, A. Hoffmann, F. Freimuth, and Y. Mokrousov, Spin Hall Effects in Metallic Antiferromagnets, Phys. Rev. Lett. 113, 196602 (2014).

[30] M. F. Jakobsen, K. B. Naess, P. Dutta, A. Brataas, and A. Qaiumzadeh, Electrical and thermal transport in antiferromagnet-superconductor junctions, Phys. Rev. B 102, 140504(R) (2020).

[31] M. M. Otrokov, I. I. Klimovskikh, H. Bentmann, D. Estyunin, A. Zeugner, Z. S. Aliev, S. Gaß, A. U. B. Wolter, A. V. Koroleva, A. M. Shikin, M. Blanco-Rey, M. Hoffmann, I. P. Rusinov, A. Yu. Vyazovskaya, S. V. Eremeev, Yu. M. Koroteev, V. M. Kuznetsov, F. Freyse, J. Sánchez-Barriga, I. R. Amiraslanov, M. B. Babanly, N. T. Mamedov, N. A. Abdullayev, V. N. Zverev, A. Alfonsov, V. Kataev, B. Büchner, E. F. Schwier, S. Kumar, A. Kimura, L. Petaccia, G. D. Santo, R. C. Vidal, S. Schatz, K. Kißner, M. Ünzelmann, C. H. Min, S. Moser, T. R. F. Peixoto, F. Reinert, A. Ernst, P. M. Echenique, A. Isaeva, and E. V. Chulkov, Prediction and observation of an antiferromagnetic topological insulator, Nature (London) 576, 416 (2019).

[32] C. Liu, Y. Wang, H. Li, Y. Wu, Y. Li, J. Li, K. He, Y. Xu, J. Zhang, and Y. Wang, Robust axion insulator and Chern insulator phases in a two-dimensional antiferromagnetic topological insulator, Nat. Mater. 19, 522 (2020).

[33] C. Niu, H. Wang, N. Mao, B. Huang, Y. Mokrousov, and Y. Dai, Antiferromagnetic Topological Insulator with Nonsymmorphic Protection in Two Dimensions, Phys. Rev. Lett. 124, 066401 (2020).

[34] R. S. K. Mong, A. M. Essin, and J. E. Moore, Antiferromagnetic topological insulators, Phys. Rev. B 81, 245209 (2010).

[35] A. Heimes, P. Kotetes, and G. Schön, Majorana fermions from Shiba states in an antiferromagnetic chain on top of a superconductor, Phys. Rev. B 90, 060507(R) (2014).

[36] A. Tan, V. Labracherie, N. Kunchur, A. U. B. Wolter, J. Cornejo, J. Dufouleur, B. Büchner, A. Isaeva, and R. Giraud, Metamagnetism of Weakly Coupled Antiferromagnetic Topological Insulators, Phys. Rev. Lett. 124, 197201 (2020).

[37] P. M. Sass, J. Kim, D. Vanderbilt, J. Yan, and W. Wu, Robust $a$-Type Order and Spin-Flop Transition on the Surface of the Antiferromagnetic Topological Insulator $\mathrm{MnBi}_{2} \mathrm{Te}_{4}$, Phys. Rev. Lett. 125, 037201 (2020).

[38] K. Machida, K. Nokura, and T. Matsubara, Theory of antiferromagnetic superconductors, Phys. Rev. B 22, 2307 (1980).

[39] M. Weides, M. Disch, H. Kohlstedt, and D. E. Bürgler, Observation of Josephson coupling through an interlayer of antiferromagnetically ordered chromium, Phys. Rev. B 80, 064508 (2009).

[40] J. W. A. Robinson, G. B. Halász, and M. G. Blamire, Crossover Induced by Spin-Density-Wave Interference in the Coherence of Singlet Electron Pairs in Cr, Phys. Rev. Lett. 103, 207002 (2009).

[41] C. Bell, E. J. Tarte, G. Burnell, C. W. Leung, D.-J. Kang, and M. G. Blamire, Proximity and Josephson effects in superconductor/antiferromagnetic $\mathrm{Nb} / \gamma-\mathrm{Fe}_{50} \mathrm{Mn}_{50}$ heterostructures, Phys. Rev. B 68, 144517 (2003).

[42] M. J. Nass, K. Levin, and G. S. Grest, Bardeen-CooperSchrieffer Pairing in Antiferromagnetic Superconductors, Phys. Rev. Lett. 46, 614 (1981).
[43] M. H. Fischer, M. Sigrist, and D. F. Agterberg, Superconductivity Without Inversion and Time-Reversal Symmetries, Phys. Rev. Lett. 121, 157003 (2018).

[44] S. Coh, M. L. Cohen, and S. G Louie, Large electron-phonon interactions from FeSe phonons in a monolayer, New J. Phys. 17, 073027 (2015).

[45] Z. F. Wang, H. Zhang, D. Liu, C. Liu, C. Tang, C. Song, Y. Zhong, J. Peng, F. Li, C. Nie, L. Wang, X. J. Zhou, X. Ma, Q. K. Xue, and F. Liu, Topological edge states in a high-temperature superconductor $\mathrm{FeSe} / \mathrm{SrTiO}_{3}(001)$ film, Nat. Mater. 15, 968 (2016).

[46] Taking a smooth transition between the superconductor and antiferromagnet does not impact the results qualitatively.

[47] Charge renormalization is reabsorbed in Eq. (2).

[48] M. P. L. Sancho, J. M. L. Sancho, J. M. L. Sancho, and J. Rubio, Highly convergent schemes for the calculation of bulk and surface green functions, J. Phys. F 15, 851 (1985).

[49] R. Jackiw and C. Rebbi, Solitons with fermion number, Phys. Rev. D 13, 3398 (1976).

[50] P. San-Jose, J. L. Lado, R. Aguado, F. Guinea, and J. FernándezRossier, Majorana Zero Modes in Graphene, Phys. Rev. X 5, 041042 (2015).

[51] J. L. Lado and M. Sigrist, Two-Dimensional Topological Superconductivity With Antiferromagnetic Insulators, Phys. Rev. Lett. 121, 037002 (2018).

[52] A. L. R. Manesco, G. Weber, and D. Rodrigues, Effective model for Majorana modes in graphene, Phys. Rev. B 100, 125411 (2019).

[53] J. L. Lado and M. Sigrist, Solitonic in-gap modes in a superconductor-quantum antiferromagnet interface, Phys. Rev. Research 2, 023347 (2020).

[54] A. H. Castro Neto, F. Guinea, N. M. R. Peres, K. S. Novoselov, and A. K. Geim, The electronic properties of graphene, Rev. Mod. Phys. 81, 109 (2009).

[55] J. C. Budich and E. Ardonne, Equivalent topological invariants for one-dimensional Majorana wires in symmetry class $d$, Phys. Rev. B 88, 075419 (2013).

[56] See Supplemental Material at http://link.aps.org/supplemental/ 10.1103/PhysRevResearch.3.L012021 for details on the microscopic mean field.

[57] Z. Qiao, S. A. Yang, W. Feng, W.-K. Tse, J. Ding, Y. Yao, J. Wang, and Q. Niu, Quantum anomalous Hall effect in graphene from Rashba and exchange effects, Phys. Rev. B 82, 161414(R) (2010).

[58] C. L. Kane and E. J. Mele, Quantum Spin Hall Effect in Graphene, Phys. Rev. Lett. 95, 226801 (2005).

[59] C. Popovici, C. S. Fischer, and L. von Smekal, Fermi velocity renormalization and dynamical gap generation in graphene, Phys. Rev. B 88, 205429 (2013).

[60] T. Stauber, P. Parida, M. Trushin, M. V. Ulybyshev, D. L. Boyda, and J. Schliemann, Interacting Electrons in Graphene: Fermi Velocity Renormalization and Optical Response, Phys. Rev. Lett. 118, 266801 (2017).

[61] The term in $\mathcal{H}^{\mathrm{MF}}$ creating the gap opening shows a similar exponential dependence.

[62] J. Bardeen, L. N. Cooper, and J. R. Schrieffer, Theory of superconductivity, Phys. Rev. 108, 1175 (1957).

[63] A. M. Lobos, R. M. Lutchyn, and S. Das Sarma, Interplay of Disorder and Interaction in Majorana Quantum Wires, Phys. Rev. Lett. 109, 146403 (2012). 
[64] N. M. Gergs, L. Fritz, and D. Schuricht, Topological order in the Kitaev/Majorana chain in the presence of disorder and interactions, Phys. Rev. B 93, 075129 (2016).

[65] İ. Adagideli, M. Wimmer, and A. Teker, Effects of electron scattering on the topological properties of nanowires: Majorana fermions from disorder and superlattices, Phys. Rev. B 89, 144506 (2014).

[66] See Supplemental Material at http://link.aps.org/supplemental/ 10.1103/PhysRevResearch.3.L012021 for details on the role of Anderson disorder and different interfaces.

[67] Although square lattice models can have antiferromagnetism, their low energy model is usually not of the Dirac form.

[68] M. McGuire, Crystal and magnetic structures in layered, transition metal dihalides and trihalides, Crystals 7, 121 (2017).

[69] L. Webster and J.-A. Yan, Strain-tunable magnetic anisotropy in monolayer $\mathrm{CrCl}_{3}, \mathrm{CrBr}_{3}$, and $\mathrm{CrI}_{3}$, Phys. Rev. B 98, 144411 (2018).

[70] Z. Wu, J. Yu, and S. Yuan, Strain-tunable magnetic and electronic properties of monolayer $\mathrm{CrI}_{3}$, Phys. Chem. Chem. Phys. 21, 7750 (2019).

[71] A. Möller, U. Löw, T. Taetz, M. Kriener, G. André, F. Damay, O. Heyer, M. Braden, and J. A. Mydosh, Structural domain and finite-size effects of the antiferromagnetic $S=1 / 2$ honeycomb lattice in $\mathrm{InCu}_{2 / 3} \mathrm{~V}_{1 / 3} \mathrm{O}_{3}$, Phys. Rev. B 78, 024420 (2008).

[72] A. A. Tsirlin, O. Janson, and H. Rosner, $\beta-\mathrm{Cu}_{2} \mathrm{~V}_{2} \mathrm{O}_{7}$ : A spin- $\frac{1}{2}$ honeycomb lattice system, Phys. Rev. B 82, 144416 (2010).

[73] T. O. Wehling, A. M. Black-Schaffer, and A. V. Balatsky, Dirac materials, Adv. Phys. 63, 1 (2014).

[74] P. Seth, P. Hansmann, A. van Roekeghem, L. Vaugier, and S. Biermann, Towards a First-Principles Determination of Effective Coulomb Interactions in Correlated Electron Materials: Role of Intershell Interactions, Phys. Rev. Lett. 119, 056401 (2017).

[75] M. Schüler, M. Rösner, T. O. Wehling, A. I. Lichtenstein, and M. I. Katsnelson, Optimal Hubbard Models for Materials with Nonlocal Coulomb Interactions: Graphene, Silicene, and Benzene, Phys. Rev. Lett. 111, 036601 (2013).
[76] I. Timrov, N. Marzari, and M. Cococcioni, Hubbard parameters from density-functional perturbation theory, Phys. Rev. B 98, 085127 (2018).

[77] I. Timrov, N. Marzari, and M. Cococcioni, Self-consistent Hubbard parameters from density-functional perturbation theory in the ultrasoft and projector-augmented wave formulations, Phys. Rev. B 103, 045141 (2021).

[78] See Supplemental Material at http://link.aps.org/supplemental/ 10.1103/PhysRevResearch.3.L012021 for a discussion on the $V_{2}$ values in two-dimensional materials.

[79] P. Törmä and K. Sengstock, Quantum Gas ExperimentsExploring Many-Body States (Imperial College Press, London, 2015).

[80] G. Jotzu, M. Messer, R. Desbuquois, M. Lebrat, T. Uehlinger, D. Greif, and T. Esslinger, Experimental realization of the topological Haldane model with ultracold fermions, Nature (London) 515, 237 (2014).

[81] A. Mazurenko, C. S. Chiu, G. Ji, M. F. Parsons, M. KanászNagy, R. Schmidt, F. Grusdt, E. Demler, D. Greif, and M. Greiner, A cold-atom Fermi-Hubbard antiferromagnet, Nature (London) 545, 462 (2017).

[82] S. Baier, M. J. Mark, D. Petter, K. Aikawa, L. Chomaz, Z. Cai, M. Baranov, P. Zoller, and F. Ferlaino, Extended BoseHubbard models with ultracold magnetic atoms, Science $\mathbf{3 5 2}$, 201 (2016).

[83] E. Guardado-Sanchez, B. M. Spar, P. Schauss, R. Belyansky, J. T. Young, P. Bienias, A. V. Gorshkov, T. Iadecola, and W. S. Bakr, Quench dynamics of a Fermi gas with strong long-range interactions, arXiv:2010.05871.

[84] B. Yan, S. A. Moses, B. Gadway, J. P. Covey, K. R. A. Hazzard, A. M. Rey, D. S. Jin, and J. Ye, Observation of dipolar spinexchange interactions with lattice-confined polar molecules, Nature (London) 501, 521 (2013).

[85] D. Mitra, P. T. Brown, E. Guardado-Sanchez, S. S. Kondov, T. Devakul, D. A. Huse, P. Schauß, and W. S. Bakr, Quantum gas microscopy of an attractive Fermi-Hubbard system, Nat. Phys. 14, 173 (2017). 